\title{
A Note on Function Space and Boundedness of the General Fractional Integral in Continuous Time Random Walk
}

\author{
Qin Fan ${ }^{1,2} \cdot$ Guo-Cheng Wu ${ }^{2} \cdot$ Hui Fu ${ }^{3}$
}

Received: 19 October 2021 / Accepted: 28 November 2021 / Published online: 10 December 2021

(C) The Author(s) 2021

\begin{abstract}
The general fractional calculus becomes popular in continuous time random walk recently. However, the boundedness condition of the general fractional integral is one of the fundamental problems. It wasn't given yet. In this short communication, the classical norm space is used, and a general boundedness theorem is presented. Finally, various long-tailed waiting time probability density functions are suggested in continuous time random walk since the general fractional integral is well defined.
\end{abstract}

Keywords General fractional integral $\cdot$ Minkowski inequality $\cdot$ Boundedness

Mathematics Subject Classification $26 \mathrm{~A} 33 \cdot 34 \mathrm{~A} 08 \cdot 35 \mathrm{R} 11$

\section{Introduction}

A fractional integral was proposed with general kernels in [1-3]

$$
I_{a^{+}}^{\alpha, g} f(t):=\int_{a}^{t} \frac{1}{\Gamma(\alpha)}(g(t)-g(s))^{\alpha-1} g^{\prime}(s) f(s) d s,
$$

Guo-Cheng Wu

wuguocheng@gmail.com

Qin Fan

mathqin@yeah.net

Hui Fu

fuhuimath@163.com

1 School of Science, Chongqing University of Posts and Telecommunications,

Chongqing 400065, People's Republic of China

2 Data Recovery Key Laboratory of Sichuan Province, College of Mathematics and Information Science, Neijiang Normal University, Neijiang 641100, People's Republic of China

3 Department of Mathematics, Harbin Institute of Technology (Weihai), Weihai 264209,

People's Republic of China 
where $\alpha$ is a positive real number, $g(t)$ is strictly increasing function on $[a, b]$ and $g(a) \geq 0$. Due to the new features in comparison with the standard fractional derivatives, much attention has been paid to theoretical research and applications, for example, fractional calculus of variations [4], the Laplace transform [5, 6], exact solution [7, 8] and numerical methods [9].

Motivated by the continuous time random walk understood by means of the standard fractional calculus $[10,11]$, suppose a long-tailed waiting time probability density function [12]

$$
\phi(t) \sim \frac{\alpha A_{\alpha}}{\Gamma(1-\alpha)(g(t)-g(a))^{\alpha+1}}
$$

which is more general than the power law function

$$
\phi(t) \sim \frac{\alpha A_{\alpha}}{\Gamma(1-\alpha)(t-a)^{\alpha+1}}
$$

Then, one comes across a general time-fractional Fokker-Planck equation with the general fractional integral

$$
P(x, t)-P(x, 0)=I_{a^{+}}^{\alpha, g}\left(K_{\alpha}\left(\frac{\partial^{2}}{\partial x^{2}}-\frac{\partial}{\partial x} \frac{F(x)}{k_{b} T}\right) P(x, t)\right), 0<\alpha \leq 1 .
$$

where $T$ is the temperature, $P(x, t)$ is the probability density function, $K_{\alpha}$ is the diffusion coefficient, $F(x)$ represents external force field and $k_{b}$ represents the Boltzmann constant. So the physical meaning of the general fractional derivative or the kernel function $g(t)$ was provided. More details were shown in [12]. However, one of the fundamental problems is still not addressed yet. That is, what is the function space of the general fractional integral and what are the constraint conditions with respect to $g(t)$ ?

A norm of the general fractional integral was recently given (see [13]). However, the condition $c=\frac{1}{p}$ and $g(t)=t$ is too strong there. For a general function $g(t) \neq t$, it cannot be reduced to the Lebesgue space $L_{p}[a, b](1 \leq p)$. In order to address this problem and improve the existing results, we reconsider the classical norm used by Kilbas et al for the Riemann-Liouville integral [3]. We present the boundedness of the general fractional integral operator in $X_{c}^{p}(a, b)$ and we give the constraint conditions of $g(t)$.

\section{General Fractional Integral}

Let us revisit the general fractional integral in $X_{c}^{p}(a, b)$ with the norm.

Definition 2.1 [3] Let $c \in \mathbb{R}, 1 \leq p \leq \infty$ and $0<a<b<\infty$. The space $X_{c}^{p}(a, b)$ is defined to consist of those complex-valued Lebesgue measurable functions on $[a, b]$ for which $\|f\|_{X_{c}^{p}}<\infty$, with 


$$
\|f\|_{X_{c}^{p}}=\left(\int_{a}^{b}\left|t^{c} f(t)\right|^{p} \frac{d t}{t}\right)^{\frac{1}{p}} \quad(1 \leq p<\infty, c \in \mathbb{R})
$$

and

$$
\|f\|_{X_{c}^{\infty}}=e s s \sup _{a \leq t \leq b}\left[t^{c}|f(t)|\right] \quad(p=\infty) .
$$

In particular, when $c=\frac{1}{p}$, the space $X_{c}^{p}(a, b)$ coincides with the space $L_{p}[a, b]$ with

$$
\|f\|_{p}=\left(\int_{a}^{b}|f(t)|^{p} d t\right)^{\frac{1}{p}} \quad(1 \leq p<\infty, c \in \mathbb{R})
$$

and

$$
\|f\|_{\infty}=e s s \sup _{a \leq t \leq b}|f(t)| \quad(p=\infty) .
$$

The following proves that the norm defined in $X_{c}^{p}(a, b)$ satisfies three axioms.

Lemma 2.2 For arbitrary $f(t)$ and $h(t) \in X_{c}^{p}(a, b), k \in \mathbb{R}$, (4) and (5) can be a norm of $X_{c}^{p}(a, b)$.

Proof For $1 \leq p<\infty$, obviously, $\|f\|_{X_{c}^{p}} \geq 0$ and $\|f\|_{X_{c}^{p}}=0$ if and only if $f=0$.

$$
\|k f\|_{X_{c}^{p}}=\left(\int_{a}^{b}\left|t^{c} k f(t)\right|^{p} \frac{d t}{t}\right)^{\frac{1}{p}}=|k|\|f\|_{X_{c}^{p}} \quad(k \in \mathbb{R}) .
$$

The trigonometric inequality is true. In fact, from Minkowski inequality $\|f+h\|_{L_{p}} \leq\|f\|_{L_{p}}+\|h\|_{L_{p}}$, we have

$$
\begin{aligned}
\|f+h\|_{X_{c}^{p}} & =\left(\int_{a}^{b}\left|t^{c}(f(t)+h(t))\right|^{p} \frac{d t}{t}\right)^{\frac{1}{p}} \\
& =\left(\int_{a}^{b}\left|t^{c-\frac{1}{p}}(f(t)+h(t))\right|^{p} d t\right)^{\frac{1}{p}} \\
& \leq\left(\int_{a}^{b}\left|t^{c-\frac{1}{p}} f(t)\right|^{p} d t\right)^{\frac{1}{p}}+\left(\int_{a}^{b}\left|t^{c-\frac{1}{p}} h(t)\right|^{p} d t\right)^{\frac{1}{p}} \\
& =\left(\int_{a}^{b}\left|t^{c} f(t)\right|^{p} \frac{d t}{t}\right)^{\frac{1}{p}}+\left(\int_{a}^{b}\left|t^{c} h(t)\right|^{p} \frac{d t}{t}\right)^{\frac{1}{p}} \\
& =\|f\|_{X_{c}^{p}}+\|h\|_{X_{c}^{p} .}
\end{aligned}
$$

Moreover, since $f(t)$ and $h(t) \in X_{c}^{p}(a, b)$ we know that $\|f\|_{X_{c}^{p}}<\infty$ and $\|h\|_{X_{c}^{p}}<\infty$. Therefore, from the above formula, we derive that $t^{c-\frac{1}{p}} f(t)$ and $t^{c-\frac{1}{p}} h(t)$ are $L_{p}$ 
integrable. Thus, the inequality (6) holds and $X_{c}^{p}(a, b)(1 \leq p \leq \infty)$ is a normed linear space.

For $p=\infty$, the norm (5) also can be easily verified.

The general fractional integral can be derived by the $n$-fold integral method from [3]. Suppose $n \in \mathbb{N}=\{1,2, \cdots\}$ and $g(t) \in C^{1}[a, b]$ is a strictly increasing function and $g(a) \geq 0$ for $t \in[a, b]$, the $n$-fold integral reads

$$
\begin{aligned}
I_{a^{+}}^{n, g} f(t): & =\int_{a}^{t} g^{\prime}\left(t_{1}\right) d t_{1} \int_{a}^{t_{1}} g^{\prime}\left(t_{2}\right) d t_{2} \cdots \int_{a}^{t_{n-1}} g^{\prime}(s) f(s) d s \\
& =\int_{a}^{t} g^{\prime}(s) f(s) d s \int_{s}^{t} g^{\prime}\left(t_{1}\right) d t_{1} \int_{s}^{t_{1}} g^{\prime}\left(t_{2}\right) d t_{2} \cdots \int_{s}^{t_{n-2}} g^{\prime}\left(t_{n-1}\right) d t_{n-1} \\
& =\frac{1}{\Gamma(n)} \int_{a}^{t}(g(t)-g(s))^{n-1} g^{\prime}(s) f(s) d s .
\end{aligned}
$$

As a result, for any positive real number $\alpha$, we give the definition general fractional integral in $X_{c}^{p}(a, b)$ space as follows.

Definition 2.3 (General fractional integral) Suppose $f(t) \in X_{c}^{p}(a, b)$ and $g(t) \in C^{1}[a, b]$ is a strictly increasing function with $g(a) \geq 0$. The general fractional integral of order $\alpha(\alpha>0)$ is defined by

$$
I_{a^{+}}^{\alpha, g} f(t):=\frac{1}{\Gamma(\alpha)} \int_{a}^{t}(g(t)-g(s))^{\alpha-1} g^{\prime}(s) f(s) d s .
$$

For some specific functions, we obtain some well known definitions of fractional integrals, for example, the Riemann-Liouville integral [3] when $g(t)=t$, the Hadamard integral when $g(t)=\ln t$ [3] and the generalized integral [14] when $g(t)=\frac{t^{\sigma}}{\sigma+1}$, the fractional integral with exponential memory [15, 16] when $g(t)=e^{\lambda t}$.

The general fractional integral operator $I_{a^{+}}^{\alpha, g}$ is bounded in the space $X_{c}^{p}(a, b)(1 \leq p \leq \infty)$. We assume $a>0$ and $g(a)>0$ for simplicity in the sequel.

Theorem 2.4 (Boundedness theorem) Let $f \in X_{c}^{p}(a, b)$ and $u=\frac{g(t)}{g(s)}$ where $a \leq s \leq t$, $1 \leq p \leq \infty, \quad c \geq \frac{1}{p}$. If there exists a function $F(u) \in C\left[1, \frac{g(b)}{g(a)}\right]$ such that $g^{-1}(u g(s)) \leq F(u) s$ and $\frac{d\left(g^{-1}(u g(s))\right)}{d s} \leq F(u)$, respectively, then

$$
\left\|I_{a^{+}}^{\alpha, g} f(t)\right\|_{X_{c}^{p}} \leq K\|f\|_{X_{c}^{p}}
$$

where

$$
K:=\int_{1}^{\frac{g(b)}{g(a)}} \frac{g(b)^{\alpha}}{\Gamma(\alpha)} \frac{(u-1)^{\alpha-1}}{u^{\alpha+1}} F(u)^{c} d u .
$$

Proof For $1 \leq p<\infty$, since $g(a)>0$, from (4) and (8), we have 


$$
\begin{aligned}
\left\|I_{a^{+}}^{\alpha, g} f(t)\right\|_{X_{c}^{p}} & =\left(\int_{a}^{b}\left|t^{c} \int_{a}^{t} \frac{1}{\Gamma(\alpha)}(g(t)-g(s))^{\alpha-1} g^{\prime}(s) f(s) d s\right|^{p} \frac{d t}{t}\right)^{\frac{1}{p}} \\
& =\left(\int_{a}^{b} d t\left|\int_{1}^{\frac{g(t)}{g(a)}} \frac{t^{c-\frac{1}{p}}}{\Gamma(\alpha)}\left(\frac{g(t)}{u}\right)^{\alpha-1}(u-1)^{\alpha-1} f\left(g^{-1}\left(\frac{g(t)}{u}\right)\right) \frac{g(t)}{u^{2}} d u\right|^{p}\right)^{\frac{1}{p}}
\end{aligned}
$$

Since $f(t) \in X_{c}^{p}(a, b)$ and $g(t) \in C^{1}[a, b]$ is a strictly increasing function, then $t^{c-\frac{1}{p}} g(t)^{\alpha} f\left(g^{-1}\left(\frac{g(t)}{u}\right)\right) \in L_{p}[a, b]$. Hence, we can apply the general Minkowski inequality [2] and give

$$
\begin{aligned}
\left\|I_{a^{+}}^{\alpha, g} f(t)\right\|_{X_{c}^{p}} \leq & \int_{1}^{\frac{g(b)}{g(a)}} \frac{1}{\Gamma(\alpha)} \frac{(u-1)^{\alpha-1}}{u^{\alpha+1}} d u\left(\int_{g^{-1}(u g(a))}^{b}\left|t^{c-\frac{1}{p}} g(t)^{\alpha} f\left(g^{-1}\left(\frac{g(t)}{u}\right)\right)\right|^{p} d t\right)^{\frac{1}{p}} \\
\leq & \int_{1}^{\frac{g(b)}{g(a)}} \frac{g(b)^{\alpha}}{\Gamma(\alpha)} \frac{(u-1)^{\alpha-1}}{u^{\alpha+1}} d u\left(\int_{g^{-1}(u g(a))}^{b}\left|t^{c-\frac{1}{p}} f\left(g^{-1}\left(\frac{g(t)}{u}\right)\right)\right|^{p} d t\right)^{\frac{1}{p}} \\
= & \int_{1}^{\frac{g(b)}{g(a)}} \frac{g(b)^{\alpha}}{\Gamma(\alpha)} \frac{(u-1)^{\alpha-1}}{u^{\alpha+1}} d u \\
& \left(\int_{a}^{g^{-1}\left(\frac{g(b)}{u}\right)}\left|\left(g^{-1}(u g(s))\right)^{c-\frac{1}{p}} f(s)\right|^{p}\left(g^{-1}(u g(s))\right)^{\prime} d s\right)^{\frac{1}{p}} .
\end{aligned}
$$

It follows from that $g^{-1}(u g(s)) \leq F(u) s, \frac{d\left(g^{-1}(u g(s))\right)}{d s} \leq F(u), u \geq 1$ and $c \geq \frac{1}{p}$ that

$$
\begin{aligned}
& \left(\int_{a}^{g^{-1}\left(\frac{g(b)}{u}\right)}\left|\left(g^{-1}(u g(s))\right)^{c-\frac{1}{p}} f(s)\right|^{p}\left(g^{-1}(u g(s))\right)^{\prime} d s\right)^{\frac{1}{p}} \\
& \leq F(u)^{c}\left(\int_{a}^{b}\left|s^{c-\frac{1}{p}} f(s)\right|^{p} d s\right)^{\frac{1}{p}}
\end{aligned}
$$

and

$$
\left\|I_{a^{+}}^{\alpha, g} f(t)\right\|_{X_{c}^{p}} \leq \int_{1}^{\frac{g(b)}{g(a)}} \frac{g(b)^{\alpha}}{\Gamma(\alpha)} \frac{(u-1)^{\alpha-1}}{u^{\alpha+1}} F(u)^{c} d u \cdot\|f\|_{X_{c}^{p}}
$$

Due to $F(u) \in C\left[1, \frac{g(b)}{g(a)}\right]$, we note that $K=\int_{1}^{\frac{g(b)}{g(a)}} \frac{g(b)^{\alpha}}{\Gamma(\alpha)} \frac{(u-1)^{\alpha-1}}{u^{\alpha+1}} F(u)^{c} d u<\infty$.

$$
\text { For } p=\infty,\left\|I_{a^{+}}^{\alpha, g} f(t)\right\|_{X_{c}^{\infty}}=e s s \sup _{a \leq t \leq b}\left[t^{c}\left|I_{a^{+}}^{\alpha, g} f(t)\right|\right] \text {. }
$$




$$
\begin{aligned}
\left|t^{c}\left(I_{a^{+}}^{\alpha, g} f(t)\right)\right| & =\left|t^{c} \int_{a}^{t} \frac{1}{\Gamma(\alpha)}(g(t)-g(s))^{\alpha-1} f(s) g^{\prime}(s) d s\right| \\
& =\left|\int_{1}^{\frac{g(t)}{g(a)}} \frac{t^{c}}{\Gamma(\alpha)}\left(\frac{g(t)}{u}\right)^{\alpha-1}(u-1)^{\alpha-1} f\left(g^{-1}\left(\frac{g(t)}{u}\right)\right) \frac{g(t)}{u^{2}} d u\right| \\
& \leq g(b)^{\alpha}\left|\int_{1}^{\frac{g(b)}{g(a)}} \frac{t^{c}}{\Gamma(\alpha)} \frac{(u-1)^{\alpha-1}}{u^{\alpha+1}} f\left(g^{-1}\left(\frac{g(t)}{u}\right)\right) d u\right| \\
& =g(b)^{\alpha}\left|\int_{1}^{\frac{g(b)}{g(a)}} \frac{\left(g^{-1}(u g(s))\right)^{c}}{\Gamma(\alpha)} \frac{(u-1)^{\alpha-1}}{u^{\alpha+1}} f(s) d u\right| \\
& \leq\left|\int_{1}^{\frac{g(b)}{g(a)}} \frac{g(b)^{\alpha}}{\Gamma(\alpha)} \frac{(u-1)^{\alpha-1}}{u^{\alpha+1}} F(u)^{c} s^{c} f(s) d u\right| \\
& \leq \int_{1}^{\frac{g(b)}{g(a)}} \frac{g(b)^{\alpha}}{\Gamma(\alpha)} \frac{(u-1)^{\alpha-1}}{u^{\alpha+1}} F(u)^{c} d u \cdot\|f\|_{X_{c}^{\infty}} .
\end{aligned}
$$

As a result, this completes the proof.

For $c=\frac{1}{p}$ in Definition 2.1, we return to the space $L_{p}[a, b]$ which is a special case of $X_{c}^{p}(a, b)$. The general fractional integral $I_{a^{+}}^{\alpha, g}$ is bounded in $L_{p}[a, b](1 \leq p \leq \infty$ , $0<a<b<\infty)$. It can be derived from Theorem 2.4.

Corollary 2.5 Let $c=\frac{1}{p}$ in $X_{c}^{p}(a, b)$ and $f(t) \in L_{p}[a, b]$, then the general fractional integral is bounded, that is

$$
\left\|I_{a^{+}}^{\alpha, g} f(t)\right\|_{p} \leq K\|f(t)\|_{p}
$$

where the constant $K$ is defined by (9).

\section{Conclusion}

We can verify that Theorem 2.4 can hold for several known fractional integrals:

- when $g(s)=s$ (The Riemann-Liouville integral [3]), we can find a function $F(u)=u$ such that $g^{-1}(u g(s)) \leq F(u) s$ and $\frac{d\left(g^{-1}(u g(s))\right)}{d s} \leq F(u)$, hence $K$ is defined by (9) where $F(u)=u$.

- when $g(s)=\ln s(s>1)$ (The Hadamard integral [3]), we also can choose $F(u)=\frac{\ln b}{\ln a} b^{u-1}$ such that those two conditions hold, hence $K$ is given where $F(u)=\frac{\ln b}{\ln a} b^{u-1}$. 
- when $g(s)=\frac{s^{\beta+1}}{\beta+1}(\beta>-1)$ (The generalized fractional integral [14]), we can use $F(u)=u^{\frac{1}{\beta+1}}$ and two constraint conditions with respect to $F(u)$ and $g(s)$ hold.

- when $g(s)=\frac{e^{\lambda s}}{\lambda}\left(\lambda \geq \frac{1}{a}\right)$ (The fractional integral with exponential memory [16]), we can take $F(u)=u$ to satisfy the conditions of Theorem 2.4.

As a result, we can conclude that the function space $X_{c}^{p}$ and the general fractional integral are well-defined when we use the classical norm (Definition 2.1). The above $g(t)$ with constraints conditions can be employed as long-tailed waiting time probability density function. On the other hand, they are well-defined in the general fractional calculus.

Open Access This article is distributed under the terms of the Creative Commons Attribution 4.0 International License (http://creativecommons.org/licenses/by/4.0/), which permits unrestricted use, distribution, and reproduction in any medium, provided you give appropriate credit to the original author(s) and the source, provide a link to the Creative Commons license, and indicate if changes were made.

Author Contributions All authors completed the paper together. All authors read and approved the final manuscript.

Funding This work is financially supported by the National Natural Science Foundation of China (NSFC) (Grant No. 62076141) and Sichuan Youth Science and Technology Foundation (Grant No. 22JCQN0197).

Availability of Data and Materials Not applicable.

Code Availibility Not applicable.

\section{Declarations}

Conflict of interest The authors declare that they have no conflict of interest.

Ethics approval Not applicable.

Consent to participate Not applicable.

Consent for publication Not applicable.

Open Access This article is licensed under a Creative Commons Attribution 4.0 International License, which permits use, sharing, adaptation, distribution and reproduction in any medium or format, as long as you give appropriate credit to the original author(s) and the source, provide a link to the Creative Commons licence, and indicate if changes were made. The images or other third party material in this article are included in the article's Creative Commons licence, unless indicated otherwise in a credit line to the material. If material is not included in the article's Creative Commons licence and your intended use is not permitted by statutory regulation or exceeds the permitted use, you will need to obtain permission directly from the copyright holder. To view a copy of this licence, visit http://creativecommons.org/licen ses/by/4.0/. 


\section{References}

1. Osler, T.J.: Leibniz rule for fractional derivatives generalized and an application to infinite series. SIAM J. Appl. Math. 18, 658-674 (1970)

2. Samko, S.G., Kilbas, A.A., Marichev, O.I.: Fractional integrals and derivatives: theory and applications. CRC Press (1993)

3. Kilbas, A.A., Srivastava, H.M., Trujillo, J.J.: Theory and Applications of Fractional Differential Equations. Elsevier Science B. V, Amsterdam (2006)

4. Almeida, R.: A Caputo fractional derivative of a function with respect to another function. Commun. Nonlinear Sci. Numer. Simulat. 44, 460-481 (2017)

5. Fahad, H.M., Rehman, M.U., Fernandez, A.: On Laplace transforms with respect to functions and their applications to fractional differential equations. Math. Method. Appl. Sci. (2021). https://doi. org/10.1002/mma.7772

6. Farad, J., Abdeljawad, T.: Generalized fractional derivatives and Laplace transform. Discrete Cont. Dyn-S 13, 709-722 (2020)

7. Restrepo, J.E., Ruzhansky, M., Suragan, D.: Explicit solutions for linear variable-coefficient fractional differential equations with respect to functions. Appl. Math. Comput. 403, 126177 (2021)

8. Fahad, H.M., Fernandez, A.: Operational calculus for Caputo fractional calculus with respect to functions and the associated fractional differential equations. Appl. Math. Comput. 409, 126400 (2021)

9. Wu, G.C., Kong, H., Luo, M., Huang, L.L.: Unified predictor-corrector method for fractional differential equations with general kernel functions (2021) (submitted)

10. Metzler, R., Barkai, E., Klafter, J.: Anomalous diffusion and relaxation close to thermal equilibrium: A fractional Fokker-Planck equation approach. Phys. Rev. Lett. 82, 3563 (1999)

11. Barkai, E., Metzler, R., Klafter, J.: From continuous time random walks to the fractional FokkerPlanck equation. Phys. Rev. E 61, 132 (2000)

12. Fu, H., Wu, G.C., Yang, G., Huang, L.L.: Continuous time random walk to a general fractional Fokker-Planck equation on fractal media. Eur. Phys. J. Spec. Top. (2021). https://doi.org/10.1140/ epjs/s11734-021-00323-6

13. Fahad, H.M., Fernandez, A., Rehman, M.U., Siddiqi, M.: Tempered and Hadamard-type fractional calculus with respect to functions. Mediterr. J. Math. 18, 143 (2021)

14. Katugampola, U.N.: New apporach to a generalized fractional integral. Appl. Math. Comput. 218, 860-865 (2011)

15. Garra, R., Giusti, A., Mainardi, F.: The fractional Dodson diffusion equation: a new approach. Ricerche Mat. 67, 899-909 (2018)

16. Fu, H., Wu, G.C., Yang, G., Huang, L.L.: Fractional calculus with exponential memory. Chaos 31, 031103 (2021) 